\title{
Downregulation of ARHGDIA contributes to human glioma progression through activation of Rho GTPase signaling pathway
}

\author{
Weiliang $\mathrm{Lu}^{1}$ - Xixi Wang ${ }^{1}$ - Jingjing Liu ${ }^{1} \cdot \mathrm{Yu} \mathrm{He}^{1} \cdot$ Ziwei Liang $^{1} \cdot \mathrm{Zijing} \mathrm{Xia}^{1}$. \\ Ying Cai ${ }^{1}$ - Liangxue Zhou ${ }^{2}$ - Hongxia Zhu ${ }^{3} \cdot$ Shufang Liang ${ }^{1}$
}

Received: 11 March 2016 / Accepted: 9 September 2016 / Published online: 10 October 2016

(C) The Author(s) 2016. This article is published with open access at Springerlink.com

\begin{abstract}
The protein ARHGDIA has been found to play distinct roles in cancer progression for several tumors. However, it remains elusive whether and how ARHGDIA plays functions in human glioma. In this study, we discovered that ARHGDIA is much downregulated in human glioma; meanwhile, its expression negatively correlates with glioma malignancy and positively relates to prognosis of glioma patients. It has independent predictive value of ARHGDIA expression level for overall survival of human glioma patients. Glioma patients with ARHGDIA-positive expression have a longer overall survival time than ARHGDIA-negative patients. Knockdown of ARHGDIA promotes cell proliferation, cell cycle progression, and cell migration due to the activation of Rho GTPases (Rac1, Cdc42, and RhoA) and Akt phosphorylation, whereas overexpression of ARHGDIA suppresses cell growth, cell cycle progression, and cell migration.
\end{abstract}

Shufang Liang

zizi2006@scu.edu.cn

Weiliang $\mathrm{Lu}$

lw11004cd@163.com

Xixi Wang

fozhu.1984@163.com

Jingjing Liu

1jj05080086@126.com

Yu He

sklbheyu@sina.com

Ziwei Liang

liangziweiguozhong@163.com

Zijing Xia

xia_zizi@126.com

Ying Cai

caiying157031@163.com
ARHGDIA is a potential prognostic marker and therapeutic target for human glioma.

Keywords ARHGDIA · Glioma · Downregulation · Rho GTPase

\section{Introduction}

Glioma is the most common brain tumor, accounting for about $45 \%$ of all brain tumors [1]. The World Health Organization (WHO) classifies gliomas based on the different histological tumor types (astrocytic, oligodendroglial, mixed oligoastrocytic, and ependymal glioma), as well as malignancy grades (I, II, III, and IV) [2]. The present treatment options of glioma contain surgery, chemotherapy, and radiotherapy or
Liangxue Zhou

liangxue_zhou@126.com

Hongxia Zhu

drhxzhu@cicams.ac.cn

State Key Laboratory of Biotherapy and Cancer Center, West China Hospital, Sichuan University and Collaborative Innovation Center for Biotherapy, No.17, 3rd Section of People's South Road, Chengdu 610041, Republic of China

2 Department of Neurosurgery, West China Hospital, Sichuan University, Chengdu 610041, Sichuan, Republic of China

3 Laboratory of Cell and Molecular Biology \& State Key Laboratory of Molecular Oncology, Cancer Institute \& Cancer Hospital, Chinese Academy of Medical Sciences, Beijing 100034, People's Republic of China 
the combination treatment of these methods, but the prognosis of malignant glioma remains very poor. The median survival is only 12 to 15 months for patients with glioblastoma and 2 to 5 years for patients with anaplastic glioma in the USA [3]. So, it is necessary to find biomarkers for early diagnosis and effective therapeutic targets to improve the prognosis for patients with human glioma [4].

The Rho GDP-dissociation inhibitors ARHGDIs (also named RhoGDIs) are important regulators of the Rho family of small GTPases which involves in cancer occurrence [5]. ARHGDIs take part in several biological processes during tumorigenesis and cancer progression. For example, the expression of ARHGDIs is altered in a variety of cancers, including breast cancer [6] and hepatocellular carcinoma [7]. Several novel therapeutic strategies are proposed for intervening in ARHGDI signaling [8]. These reports indicate that ARHGDI signaling may be targets for cancer therapy.

ARHGDIA is one member of ARHGDIs, which is ubiquitously expressed and interacts with several Rho GTPases, mainly including RhoA, Rac1, and Cdc42 [9]. As a regulator of Rho GTPase activity, ARHGDIA has attracted increasing attentions. Previous studies have indicated the aberrant expression of ARHGDIA is associated with cancers [6,7], whereas there is no research in detail on glioma. So our investigation aim is to explore ARHGDIA functions in glioma development. Our analyses reveal that ARHGDIA is frequently downregulated in human glioma tissues and it is significantly associated with tumor malignancy degree.

\section{Materials and methods}

\section{Glioma patients and tissue samples}

This study was approved by the Institutional Ethics Committee of State Key Laboratory of Biotherapy, West China Hospital of Sichuan University. A total of 73 glioma patients were enrolled in our study. The clinical information of patients was summarized in Table 2. The patients did not receive any preoperative cancer treatment, and their followup data were available. They were followed-up since the surgical treatment until May 2015, with a median follow-up of 20.4 months (range 0.2-68 months). During the follow-up, patients were monitored every $2-3$ months by clinic interview or phone call.

Seventy-three pairs of human glioma tissues (HGTs) and patients' autologous para-cancerous brain tissues (PBTs) were surgically resected to collect with patient's informed consent in West China Hospital, Sichuan University (Chengdu, P. R. China).

\section{Immunohistochemical analysis}

The tissues paraformaldehyde-fixed and paraffin-embedded were cut into sections of $5 \mu \mathrm{m}$ thickness for hematoxylineosin (HE) and immunohistochemistry (IHC) analysis mainly according to our previous protocols [10]. The anti-ARHGDIA antibody (Santa Cruz Biotechnology, 1:100) was used to detect the protein expression in human glioma and non-cancer tissues. Finally, the tissue slices were visualized by the 3,3diaminobenzidine solution and nuclei were slightly counterstained with hematoxylin. Substitution of the primary antibody with phosphate-buffered saline was served as a control for IHC. The intensity and percentage of positive cells were evaluated in at least five separate fields at $\times 400$ magnification.

The staining intensity was scored as 0 (negative), 1 (weak), 2 (moderate), or 3 (strong). The extent of staining was scored based on the percentage of positive tumor cells: 0 (negative), 1 (1-25\%), 2 (26-50\%), 3 (51-75\%), and 4 (76-100\%) [11, 12]. The final score of each sample was assessed by summarization of the results of the intensity and extent of staining. Therefore, each case was considered negative if the final score was $0(-)$ and positive if the final score was $1-2$ (weak, + ), more than 2 (strong, ++ ), respectively. The scoring was determined independently by two senior pathologists.

\section{Association analysis of ARHGDIA expression with glioma clinical information}

The relationship between ARHGDIA expressions with glioma clinical information was assessed based on IHC data of glioma tissues using Pearson's $\chi^{2}$ test. The clinical information included glioma patient's gender, age, tumor grades (seen in the Table 2), and patient prognosis. Among 73 glioma patients, only 37 patients whose follow-up time was more than 5 years, and the follow-up time of other 36 glioma patients was less than 5 years but all more than 3 years. The patient overall survival (OS) was evaluated using the Kaplan-Meier method. The 73 glioma patients were grouped into two groups based on the protein expression level, including ARHGDIA-negative expression $(n=32)$ and ARHGDIA-positive expression $(n=41)$. The group differences were assessed using the log-rank test.

\section{Cell culture}

Human glioma cell lines H4 and U87 were ordered from American Type Culture Collection (Manassas, VA), and U251 were ordered from the Type Culture Collection of the Chinese Academy of Sciences (Shanghai, China). Cells were cultured in DMEM medium containing $10 \%$ fetal bovine serum (FBS) (16000-044, Gibco), with $100 \mathrm{U} / \mathrm{ml}$ penicillin and $100 \mu \mathrm{g} / \mathrm{ml}$ streptomycin. Cells were incubated in $37{ }^{\circ} \mathrm{C}$ with $5 \% \mathrm{CO} 2$ and $95 \%$ air. 


\section{ARHGDIA-specific siRNAs, expression plasmid, and cell transfection}

In order to reduce the off-target effects of single siRNA, three different siRNAs against ARHGDIA had been designed and synthesized to perform the ARHGDIA knockdown experiments. One of siRNAs, siRNA1 against ARHGDIA, was synthesized based on one previous reported paper [13]. The other two siRNAs, siRNA2 and siRNA3, were designed according to our bioinformatics analysis and synthesized by the RiboBio company (RiboBio, Guangzhou, China). ARHGDIA-specific siRNA1 sequences were designed as follows: 5'UCAAUCUUGACGCCUUUCC- 3 '. The siRNA2 and siRNA3 sequences were respectively designed as following: (siRNA2) 5'-GAGCACTCGGTCAACTACA-3' and (siRNA3) 5'-GGTGTGGAGTACCGGATAA-3'. The nontargeting control siRNA oligonucleotides were 5'-UUC UCC GAA CGU GUC ACG U-3.

In order to observe cell growth and Akt signaling under ARHGDIA knockdown, $100 \mathrm{nM}$ ARHGDIA-specific siRNA was respectively transiently transfected into glioma cells for one well of a 6-well plate for $48 \mathrm{~h}$ culture using the INTERFERin transfection reagent (Polyplus Transfection).

The ARHGDIA cDNA (gi 669033301) was cloned into the expression vector pcDNA3.1-HA to obtain the recombinant plasmid pHA-ARHGDIA. The overexpression of ARHGDIA by transfection of pHA-ARHGDIA plasmids into U87 cells was performed to detect its effects on cell growth and migration. Two-microgram plasmids were transfected for each well of a 6-well plate with the reagent Lipofectamine 2000 (Cat. 11668-019, Life Technologies).

\section{Quantitative RT-PCR}

To compare endogenous gene expression in HGTs versus PBTs, RNA samples were prepared from HGTs or PBTs using TRIZOL reagent (Cat. \#15596-026, Invitrogen). First-strand complementary DNA (cDNA) synthesis was carried out with the cDNA synthesis kit (Cat. \#170-8891, Bio-Rad). PCR reaction was carried out with first-strand cDNA and one set of specific primers. For each primer set, two or three cycle numbers were tested to confirm that PCR product accumulates within a linear range. The GAPDH was amplified as a control marker with specific primers. The relative RNA expression was calculated with the comparative CT method, which was normalized to the internal references. The RT-PCR primers for ARHGDIA were designed as follows: forward primer 5'CCTCACACTGCCCCAGAGGAT-3' and reverse primer 5'GCGCACTTCTGAGCAGGAGT-3' [14]. The forward primer 5'- TGG AAG GAC TCA TGA CCA CA-3' and reverse primer 5'- TTC AGC TCA GGG ATG ACC TT-3' for the control GAPDH.

\section{Western blot}

Cell pellets were harvested after being transfected with ARHGDIA-specific siRNAs or the pHA-ARHGDIA plasmids for $48 \mathrm{~h}$. Cells were lysed to extract proteins with RIPA buffer (50 mM Tris base, $1.0 \mathrm{mM}$ EDTA, $150 \mathrm{mM}$ $\mathrm{NaCl}, 0.1 \%$ SDS, $1 \%$ Triton $\mathrm{X}-100,1 \%$ sodium deoxycholate, and $1 \mathrm{mM}$ PMSF). Proteins were separated on $12 \%$ SDS-PAGE, transferred to polyvinylidene difluoride (PVDF) membrane to incubate with rabbit antiARHGDIA antibody (1:100, Santa Cruz Biotechnology) at $4{ }^{\circ} \mathrm{C}$ overnight. A secondary antibody incubation was performed with horseradish peroxidase (HRP)-tagged antigoat (Santa Cruz) or HRP tagged anti-rabbit (Invitrogen) antibody. The PVDF membrane was re-probed with mouse anti-GAPDH antibody (Abcam) for normalization of signal. Detection was performed with Western blot reagent ECL (Amersham Biosciences).

\section{Pull-down analysis of active Cdc42/Rac1 and RhoA}

The pull-down procedures were performed based on the commercial antibody against the active form of Cdc42, Rac1, and RhoA (MuCyte Biotechnology, Nanjing, China). Cell pellets from U87 cells transfected with ARHGDIA-specific siRNA1 or pHA-ARHGDIA plasmids for $48 \mathrm{~h}$ were collected and resolved in $1 \mathrm{ml}$ of ice-cold $1 \times$ assay/lysis buffer for $10 \mathrm{~min}$. Protein supernatant was obtained by centrifugation with $14,000 \times g$ for $10 \mathrm{~min}$ at $4{ }^{\circ} \mathrm{C}$. Fifty microliters slurry of glutathione resin was washed with $1 \times$ assay/lysis buffer, and $60 \mu \mathrm{l}$ of GSTRBD (Rho-binding domain ) or $20 \mu 1$ GST-PBD(p21-binding domain) was added to bind with the resin on ice for pull-down active RhoA, Rac1, or Cdc42, respectively. Then $1 \mathrm{~mL}$ protein solution was added to incubate with the antibody-combined resin at $4{ }^{\circ} \mathrm{C}$ for $1 \mathrm{~h}$ with gentle agitation. The supernatant was removed by $7200 \times g$ centrifugation for $1 \mathrm{~min}$, and the resin was washed three times with $0.5 \mathrm{ml}$ of $1 \times$ assay/lysis buffer, suspended in $30 \mu \mathrm{l}$ of $2 \times$ reducing SDS-PAGE sample buffer to pull down the target proteins by boiling for $5 \mathrm{~min}$. The sample was separated on $12 \%$ SDS-PAGE to detect the target proteins by Western blot. The first antibodies included rabbit monoclonal antibody against RhoA (1:1000, Sion Biological), mouse monoclonal antibody against $\mathrm{Cdc} 42$ (1:1000, Abcam), and mouse monoclonal against Rac1 (1:1000; Abcam).

\section{Cell proliferation}

Cell proliferation was measured using the Cell Counting Kit-8 (CCK-8) assay (cat.ZP328-3, ZOMANIO). Three thousand cells were plated into each well of a 96-well plate after 
Fig. 1 ARHGDIA is

downregulated in human glioma. A ARHGDIA is detected in three randomly chosen HGTs via realtime PCR and western blot analysis. The transcript levels were normalized to GAPDH.

Data are presented as the mean and standard deviation (SD) of three independent experiments. B Immunohistochemical staining of ARHGDIA in HGTs and PBTs.

Tissue hematoxylin-eosin staining $(a-d)$; ARHGDIAnegative $(e)$ and ARHGDIApositive expression $(f)$ in HGTs, ARHGDIA-negative $(g)$ and ARHGDIA-positive expression (h) in PBTs. C ARHGDIA expression in different histological glioma tissues. $P A$ : pilocytic astrocytoma, $D A$ : diffuse astrocytoma, $A A$ : anaplastic astrocytoma, $G B M$ : glioblastoma. Scale bar represents $50 \mu \mathrm{m}$ (original magnification $\times 400$ ). HGTS: human glioma tissues; $P B T$ : para-cancerous brain tissues. $* * *$ means $p<0.01, * * * *$ means $p<0.001$
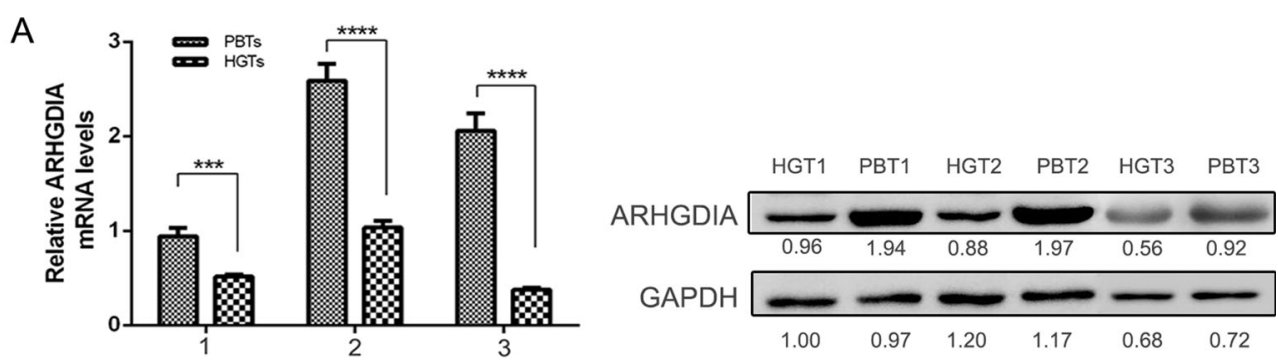

B
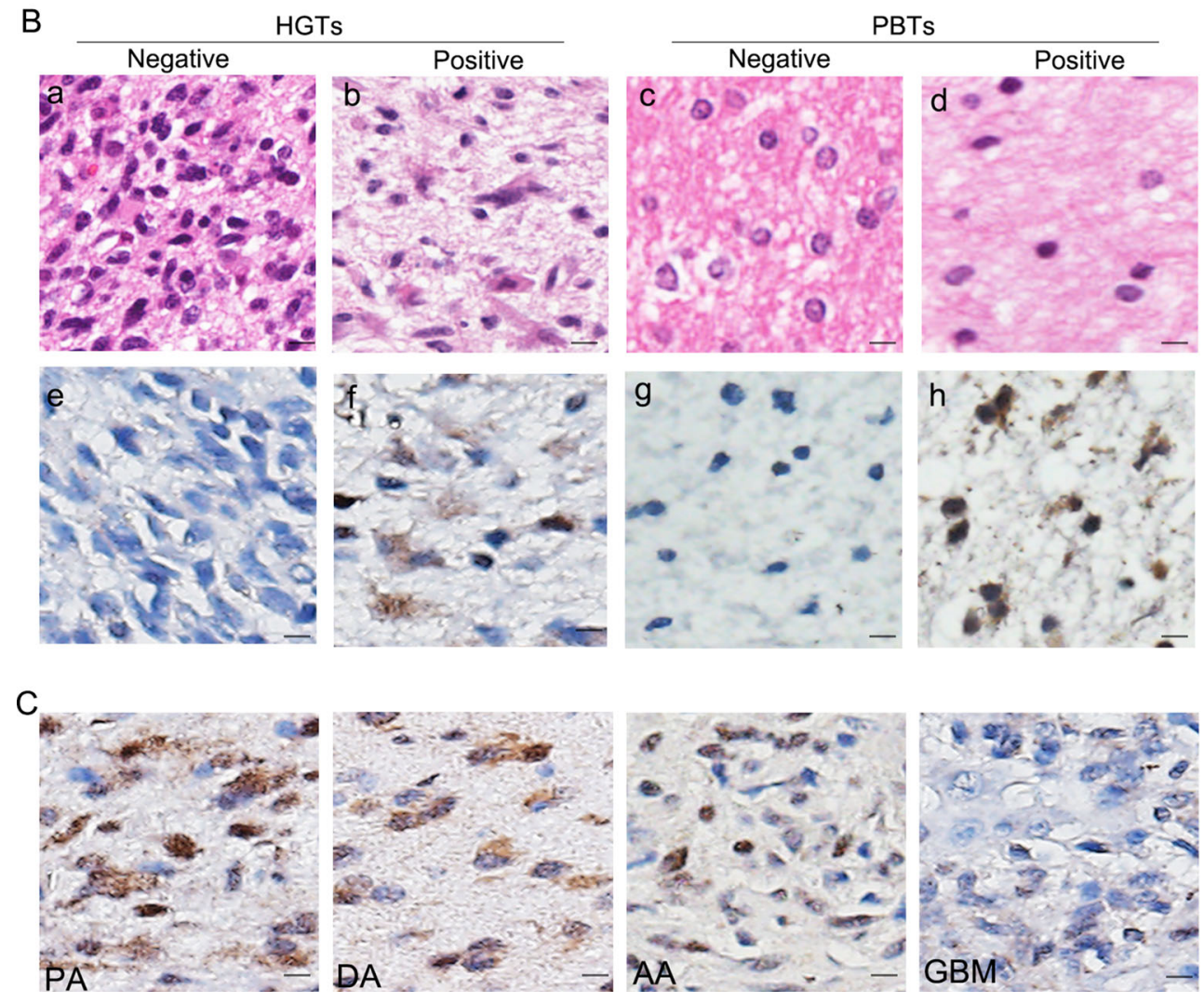

transfection with pHA-ARHGDIA plasmids or ARHGDIAspecific siRNA1 for $48 \mathrm{~h}$, in which $10 \mu \mathrm{l} \mathrm{CCK}-8$ reagents was added to $90 \mu \mathrm{l}$ of culture medium. Cells were subsequently incubated for $2 \mathrm{~h}$ at $37^{\circ} \mathrm{C}$ and the absorbance was measured at $450 \mathrm{~nm}$ on Multiskan MK3 (Thermo Scientific). Three independent experiments were performed.

\section{Flow cytometry}

For cell cycle analysis, cells were harvested after siRNA1 treatment for $48 \mathrm{~h}$, fixed in $70 \%$ ethanol on ice and stained with propidium iodide in phosphate-buffered saline containing RNase for 15 min at $37^{\circ} \mathrm{C}$ in the dark. The DNA contents

Table 1 ARHGDIA immunoreactivity between HGTs and PBTs

\begin{tabular}{ccccc}
\hline Immuno -reactivity & HGTs $(n=73)$ & & PBTs $(n=13)$ & $p$ value \\
& Percentage & Score & Percentage & $0(-)$ \\
Negative & $34.2 \%(25 / 73)$ & $0(-)$ & $15.4 \%(2 / 13)$ & $p<0.05$ \\
Positive & $65.8 \%(48 / 73)$ & $1.396 \pm 0.08(+)$ & $84.6 \%(11 / 13)$ & $2.375 \pm 0.42(++)$ \\
\hline
\end{tabular}

$P$ value was calculated the difference between ARHGDIA-positive HGTs and PBTs using Student's $t$ test. $P<0.05$ was considered statistically significant

$H G T s$ : human glioma tissues, $P B T s$ : para-cancerous brain tissues

-: negative; +: weak expression; ++: strong expression 
Table 2 Associations of ARHGDIA expression with the clinical features of glioma patients $(n=73)$

\begin{tabular}{llllll}
\hline $\begin{array}{l}\text { Clinicopathologic } \\
\text { variables }\end{array}$ & \multicolumn{2}{c}{ ARHGDIA expression } & Average score & Expression level & $p$ value \\
Gender & Negative $(n=24)$ & Positive $(n=49)$ & & \\
Male & 9 & 30 & $1.42 \pm 0.10$ & + & 0.081 \\
Female & 15 & 19 & $1.35 \pm 0.15$ & + & \\
Age, (years) & & & & \\
$\leq 56$ & 10 & 31 & $1.46 \pm 0.11$ & + & 0.131 \\
$>56$ & 14 & 18 & $1.28 \pm 0.11$ & + & \\
TNM stage & & & & \\
I-II & 11 & 9 & $1.67 \pm 0.23$ & + \\
III-IV & 12 & 33 & $1.27 \pm 0.08$ & + \\
Unknown & 1 & 7 & & & \\
\hline
\end{tabular}

+: ARHGDIA staining was scored 1-2 (weak expression) were analyzed by flow cytometry (NovoCyte, ACEA Biosciences).

\section{In vitro migration assays}

Cell migration was performed using our previous method [11]. The $1 \times 10^{4}$ cells were added into the upper chamber of the insert with the non-coated membrane (Millipore, 8-mm pore size). Cells were plated in serum-free medium, and medium containing $10 \%$ FBS in the lower chamber served as chemo-attractant. After $24 \mathrm{~h}$ of incubation, cells that did not migrate through pores were carefully wiped out with cotton swab. Cells on the lower surface of the membrane were fixed with methanol and stained with Giemsa (cat. C0121, Beyotime). Images were captured using an inverted microscope (Olympus), and the migrated cells were counted manually. Each experiment was performed in triplicates.

\section{Statistical analysis}

Statistical analysis was performed and values were expressed as the mean \pm standard deviation. The differences between groups were analyzed using Student's $t$ test (only two groups) or one-way analysis of variance (more than two groups were compared). $P<0.05$ was considered statistically significant.

\section{Results}

\section{ARHGDIA downregulation in glioma tissues}

The low expression status of ARHGDIA at mRNA and protein levels was detected in HGTs. Compared with the expression level in PBTs, ARHGDIA was greatly decreased in glioma tissues both at mRNA and protein levels (Fig. 1A). Moreover, the decreased expression of ARHGDIA was validated in 73 cases of HGTs compared with 13 PBTs by IHC (Table 1). As results, more than one thirds of human glioma tissues ( $n=25,34.2 \%$ ) had ARHGDIA-negative expression, and other 48 cases $(65.8 \%, 48 / 73)$ showed weak expression of ARHGDIA in cytoplasm (Fig. 1B-f), with mean staining scores $1.396 \pm 0.08$. While in $84.6 \%$ (11/13) PBTs, ARHGDIA was detected with a higher expression level with an average staining score $2.375 \pm 0.42$ (Fig. 1B-h). Generally, a lower expression of ARHGDIA widely exists in gliomas than that in noncancerous brain tissues $(p<0.05)$.

\section{ARHGDIA downregulation correlates with tumor stage and patient survival}

In order to evaluate whether ARHGDIA is a potential diagnosis or prognosis factor in clinical test, we analyzed the relationship between the expression of ARHGDIA and the clinicopathologic features of human glioma patients. The expression levels of ARHGDIA in glioma exhibit a tumor

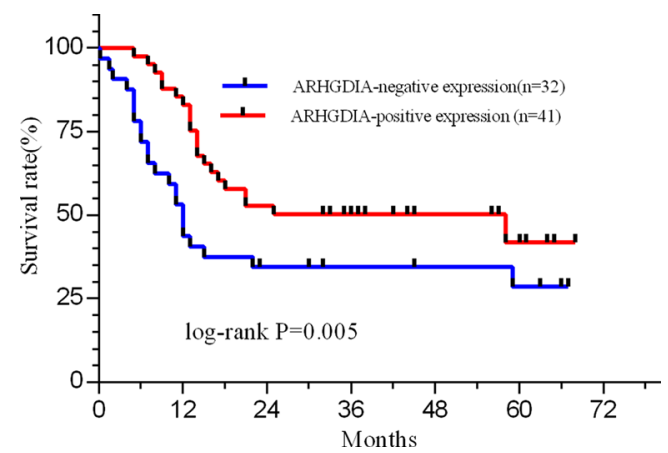

Fig. 2 Association of ARHGDIA expression and overall survival of glioma patients. The overall survival has highly significant differences between ARHGDIA-positive patients $(n=41)$ and ARHGIDA-negative expresses $(n=32)(p<0.05$ by the log-rank test). The 1-, 3-, and 5-year OS rates of the patients with low level were $39.8,30.1$, and $26.7 \%$, respectively, which were significantly lower than those with high level group (78.6, 52.4, and $44.8 \%$, respectively; $p=0.005$ ). Patients with low level of ARHGDIA have a worse postoperative overall survival 
pathological grade-dependent pattern (Table 2, $p=0.048$ ). And a significantly relative stronger ARHGDIA exists in high differentiated glioma (TNM stage I and II) than in low differentiation tumors (TNM stage III and IV) (Table 2). Moreover, ARHGDIA expression differs in different histological gliomas, including pilocytic astrocytoma, diffuse astrocytoma, anaplastic astrocytoma, and glioblastoma (Fig. 1C). But the expression level of this protein has no relationship with patient's gender and age (Table 2).

Furthermore, to determine the relationship between protein expression and $\mathrm{OS}$ of patients, 73 glioma patients were grouped into two groups, including ARHGDIA negative $(n=32)$ and positive ( $n=41)$ expression. The ARHGDIA expression level is associated with prognosis of 74 glioma patients whose follow-
Fig. 3 ARHGDIA

downregulation promotes cell proliferation in vitro. a

ARHGDIA expression in

different human glioma cell lines.

b-d The interference effect of

three siRNAs in H4, U251, and

U87 cells. e-f ARHGDIA

knockdown in $\mathrm{H} 4$ (e) or U251

cells (f) by siRNA1 treatment for

$48 \mathrm{~h}$ promotes cell proliferation

by decreasing proportion of G2/

M-phase cells $(* p<0.05)$.

siControl: nontargeting control siRNA. g The overexpression of ARHGDIA in U87 cells with transfection of pHA-ARHGDIA plasmids for $48 \mathrm{~h}$ inhibits cell proliferation by increasing the proportion of G2/M-phase cells $(* p<0.05)$
A

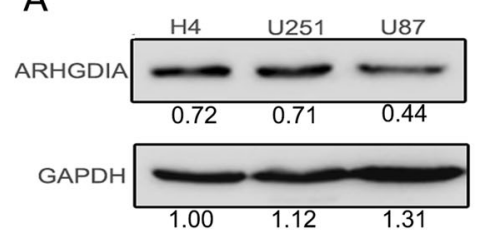

C
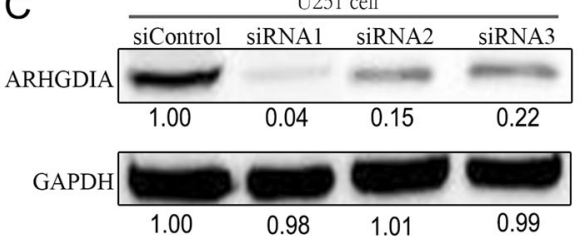

E
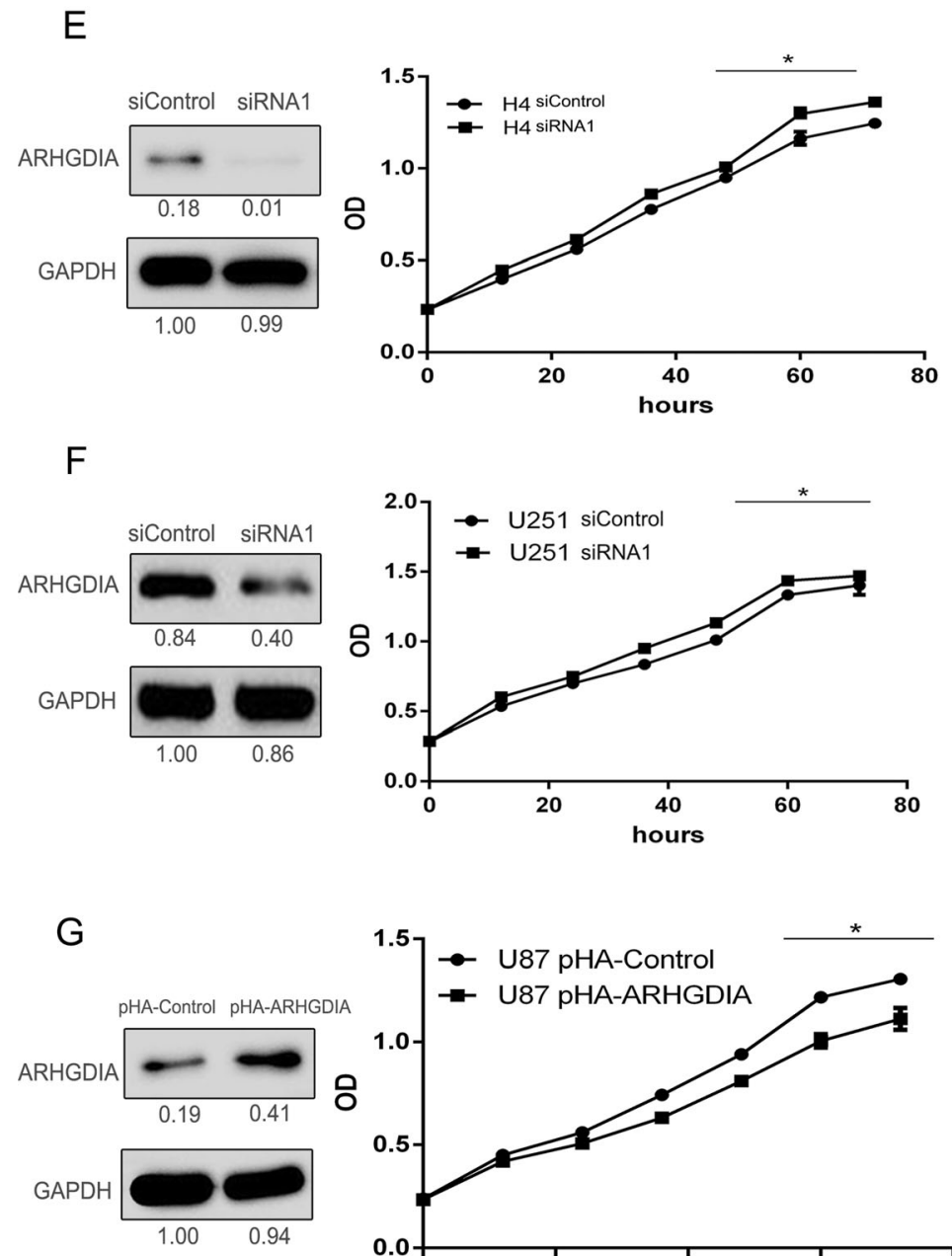
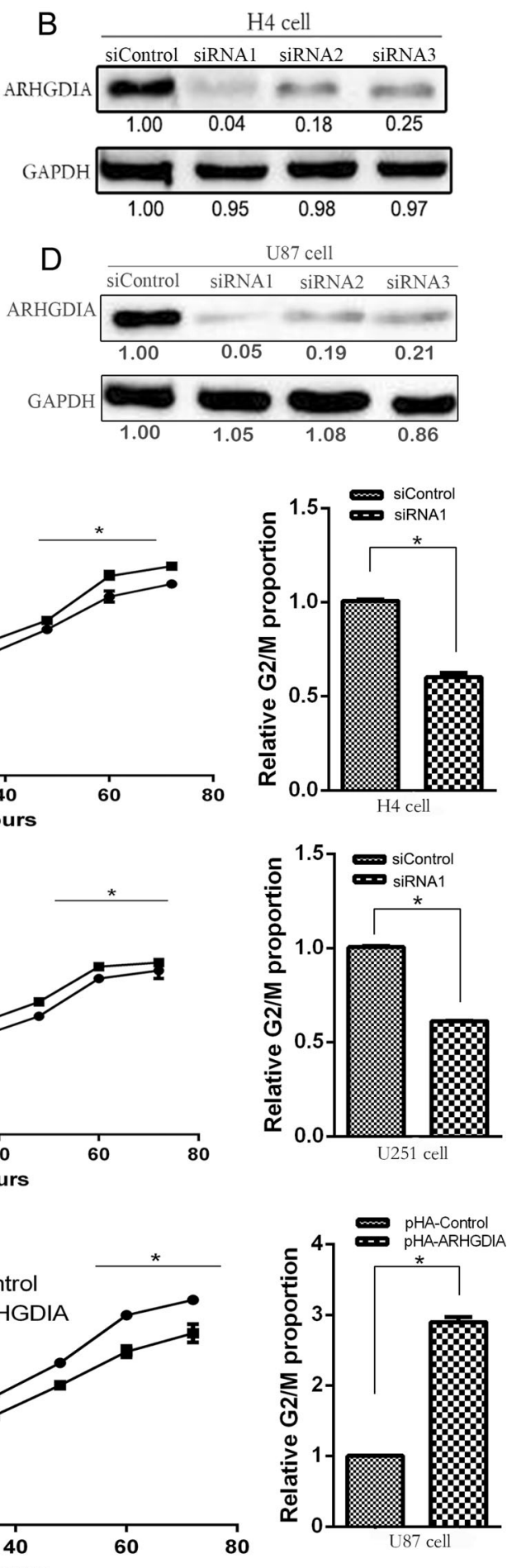

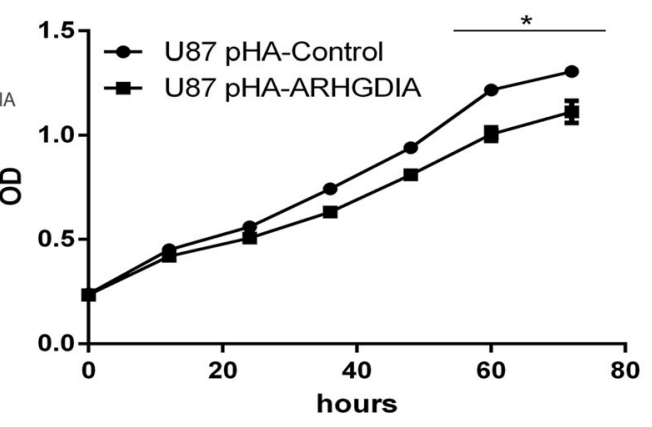
. 
up time more than 3 years. The Kaplan-Meier estimates showed significant differences in OS rates between ARHGDIA-negative patients and those with ARHGDIA-positive expression ( $P=0.005$ by the log-rank test; Fig. 2). The 32 patients with ARHGDIA-negative expression had a worse postoperative OS compared to the 41 cases of ARHGDIA-positive group $(p=0.005)$. The median survival in 41 ARHGDIA-positive patients was 25.0 months, whereas the median survival of ARHGDIA-negative patients was just 11.0 months. On the other hand, the 1-, 3-, and 5-year OS rate of the patients with the protein negative expression was $39.8,30.1$, and $26.7 \%$, respectively. Whereas the 1-, 3-, and 5-year OS rate of patients with the protein positive expression was $78.6,52.4$, and $44.8 \%$, respectively. All these data mean that patients with ARHGDIApositive expression have a longer overall survival. Generally, ARHGDIA expression level is associated with tumor grade, survival, and prognosis of glioma patients.

\section{ARHGDIA expression regulates cell proliferation and cell cycle}

We detected the endogenous expression level of ARHGDIA in three different malignant glioma cell lines, including H4,
U251 and U87 cells. The expression level of ARHGDIA in $\mathrm{H} 4$ and U251 has no obvious difference, but this protein is expressed in a relatively low level in U87 cells (Fig. 3a). We further explored this protein effects on cell growth and cell biological process by ARHGDIA knockdown in H4 cell, U251 cells and ARHGDIA overexpression in U87 cells.

The three different siRNAs had obvious interference effects for the target gene ARHGDIA expression in H4, U251, and U87 cells (Fig. 3b-d). The siRNA1 against ARHGDIA had the most effective inhibition for the protein. The inhibitory efficiency of $100 \mathrm{nM}$ specific siRNA1 was up to $90 \%$ at protein expression in these glioma cells. So we further measured cell growth responsive to siRNA1 treatment. ARHGDIA knockdown with siRNA1 significantly improved cell proliferation rate by decreasing cell proportion of $\mathrm{G} 2 / \mathrm{M}$ phase in $\mathrm{H} 4$ and $\mathrm{U} 251$ cells (Fig. 3e, f).

On the contrary, when the ARHGDIA protein is overexpressed to two times after being transfected with pHA-ARHGDIA plasmids in U87 cells, cell proliferation rate obviously decreased by increasing the proportion of G2/Mphase cells (Fig. 3g). The results suggest that ARHGDIA downregulation promotes glioma cell proliferation by regulating cell cycle distribution.
Fig. 4 ARHGDIA knockdown promotes cell migration in $\mathrm{H} 4$ and U251 cells $(\mathbf{a}-\mathbf{b})$. While ARHGDIA overexpression in U87 cells inhibits cell migration (c). NC: nontargeting control siRNA or pHA empty vector. $* p<0.05$
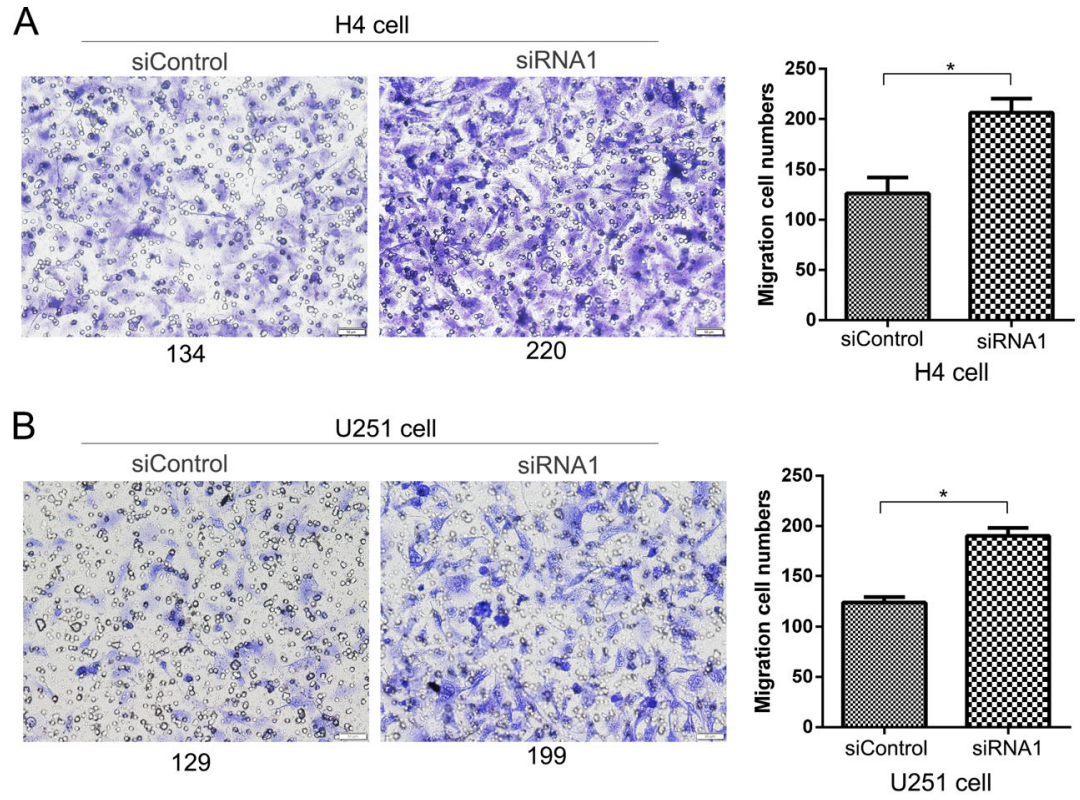

C
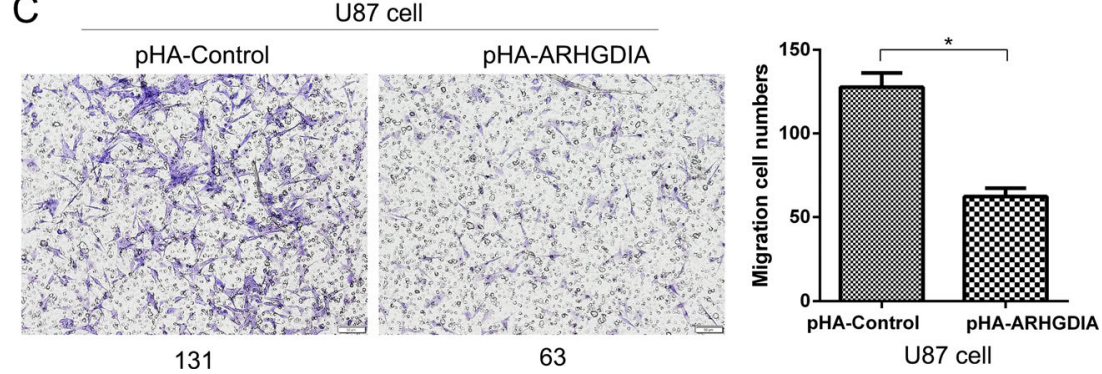


\section{ARHGDIA downregulation promotes glioma cell migration}

Next, cell migration was further analyzed to know if ARHGDIA has a crucial role in glioma cell migration ability. When ARHGDIA was knockdown by siRNA1 in H4 and U251 cells, the average number of migrated cells was significantly increased to near twofold in comparison with those by nonspecfic siRNA treatment $(P<0.05$, Fig. $4 \mathrm{a}, \mathrm{b})$. This indicated that the migration potential of human glioma cells was markedly promoted after knockdown of ARHGDIA. Conversely, ARHGDIA overexpression in U87 cells resulted in low ability of cell migration (Fig. 4c).

In conclusion, the ARHGDIA downregulation may be an important contributor to cell migration, and the expression level of ARHGDIA affects the metastatic behavior of human glioma cell lines.

\section{Downregulation of ARHGDIA increases activity of Rho GTPases}

ARHGDIs regulate the cytosol-membrane cycling of the Rho GTPase, which has a major role in controlling Rho GTPase activity and function [5]. Due to the important role of ARHGDIA in the regulation of Rho GTPase, then how does its decreased expression regulate its effector proteins including Rac1/cdc42 and RhoA in glioma cells? Based on the above conclusion of lower ARHGDIA contribution to cell growth and cell migration, we conducted pull-down assays to determine the GTP status of Rac1/cdc42 and RhoA in U87 cells after being transfected with siRNA1 and pHAARHGDIA plasmids in triplicates. As expected, the lower expression of ARHGDIA significantly induced the activation of Rac1, Cdc42, and RhoA with GTP status in U87 cells treated with ARHGDIA siRNA1 (Fig. 5a). In response to ARHGDIA knockdown, the GTPase activity of Cdc42 and RhoA was averagely increased to 2.63- and 5.50-folds, respectively, and the Rac1 was also elevated to nearly twofolds (Fig. 5b). But the higher expression of ARHGDIA significantly suppressed the active state of Rac1, Cdc42, and RhoA with GTP status in U87 cells (Fig. 5c). Responsive to influence of ARHGDIA overexpression, the GTPase activity of Cdc42 and Rac1 was averagely decreased to 1.89- and 1.92folds, and the GTPase activity of RhoA almost had almost no detection level (with average 6.8-folds decrease)(Fig. 5d). So we concluded that it has a significant role in the Rho GTPase signaling pathway by perturbations of ARHGDIA. Combined with the above conclusion of ARHGDIA downregulation in glioma cell migration, our studies confirmed that activation of signaling of Rho GTPases induced by silencing ARHGDIA contributes to cancer progression and metastasis for glioma.

\section{Downregulation of ARHGDIA increases Akt phosphorylation}

It had indicated that the GTPases of the Rho family control some major components of cellular signal transduction including Akt signaling, and inhibition of these GTPases leads to a decrease in Akt activity [15]. In this study, we also found that the phosphorylation of Akt (p-Akt) was high in human glioma cell lines, and the more higher degree of malignancy, the expression level of p-Akt was more higher (Fig. 6a). At the same time, we determined the expression level of p-Akt in HGTs and PBTs, the p-Akt expression was significantly increased in HGTs than PBTs (Fig. 6b). Combined with the ARHGDIA expression in HGTs, the above results suggest that the p-Akt expression may play an important role in the human glioma.

In order to explore whether the ARHGDIA expression had effects on p-Akt level, the phosphorylation level of Akt was
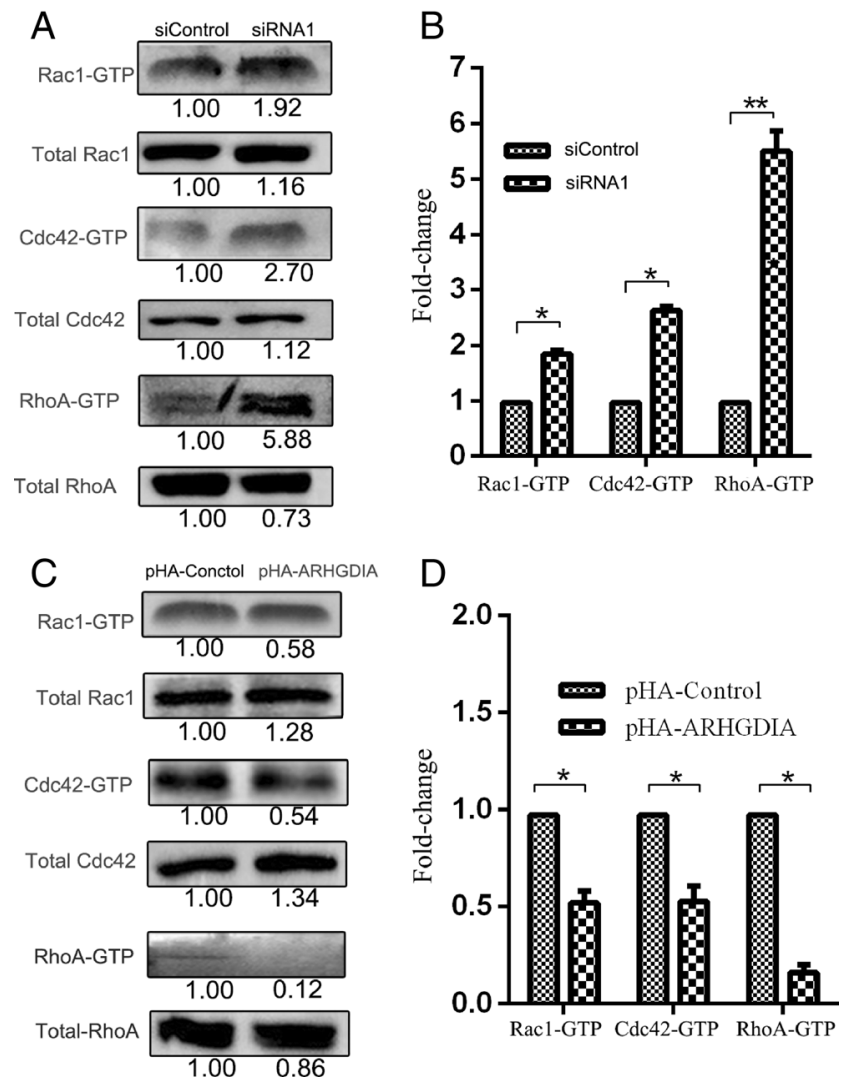

Fig. 5 ARHGDIA knockdown (a) or overexpression (c) affects the GTPase activity of Rho family proteins. Rac1, Cdc42, and RhoA with GTP form represents the GTPase activity, which was analyzed from cell lysis after enrichment by pull-down. Total target proteins (Rac1, Cdc42, and RhoA) were present in whole cell lysate. The integrated density measurements were calculated based on the fold change of active-form protein versus total target protein. The gray value of control treatment (NC) was set as 1 . The immunoblot quantitation of GTPase activity was shown in (b) and (d). NC nontargeting control siRNA or pHA empty vector. $* p<0.05, * * p<0.01$ 
Fig. 6 The Akt phosporylation level in human glioma cell lines (a, c) and in 3 randomly chosen HGTs $(\mathbf{b}, \mathbf{d})$. The 3 randomly chosen HGTs were the same samples used in the Fig. 1. The Akt phosporylation level was correlative with ARHGDIA expression in U87 cells and HGTs. siControl and pHAcontrol were the nontargeting control siRNAs or the empty transfection vector. pHAARHGDIA was ARHGDIAoverexpressing plasmid. $p-A K T$ was the phosphorylated AKT

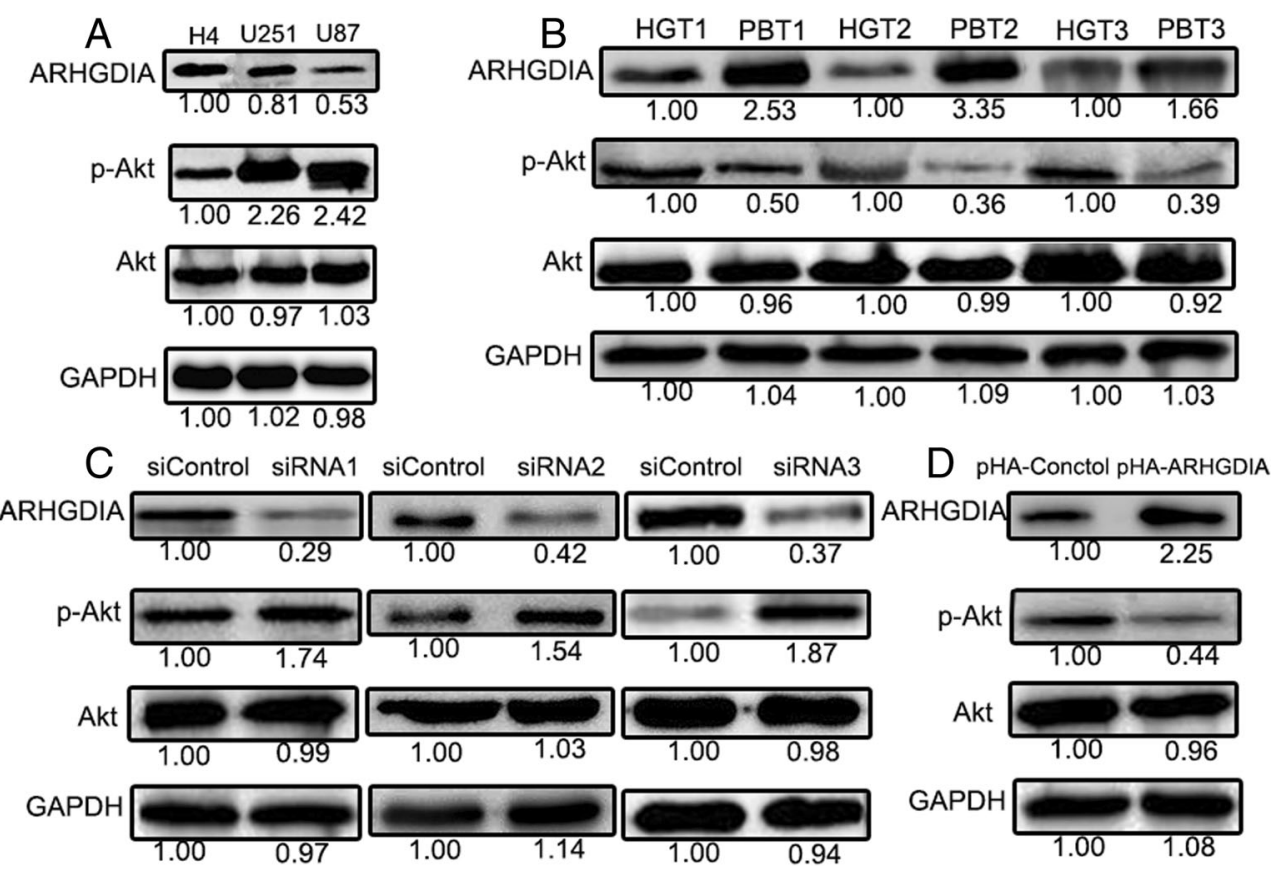

further examined by Western blot after overexpression or knockdown of ARHGDIA in U87 cells. In order to reduce the off-target effects of single siRNA, we measured Akt phosphorylation changes responsive to ARHGDIA knockdown with three siRNAs (siRNA1, siRNA2, and siRNA3) treatment. The Akt phosphorylation was respectively upregulated to 1.63-, 1.78-, and 2.34-folds in U87 cells by the siRNA1, siRNA2, and siRNA3 incubation for $48 \mathrm{~h}$ compared with the nontargeting siRNA treatment ( Fig. 6c). On the contrary, Akt phosphorylation was decreased to 2.27-folds in ARHGDIAoverexpressing U87 cells (Fig. 6d). Therefore, the downregulation of ARHGDIA regulates GTPase activity of Cdc42,

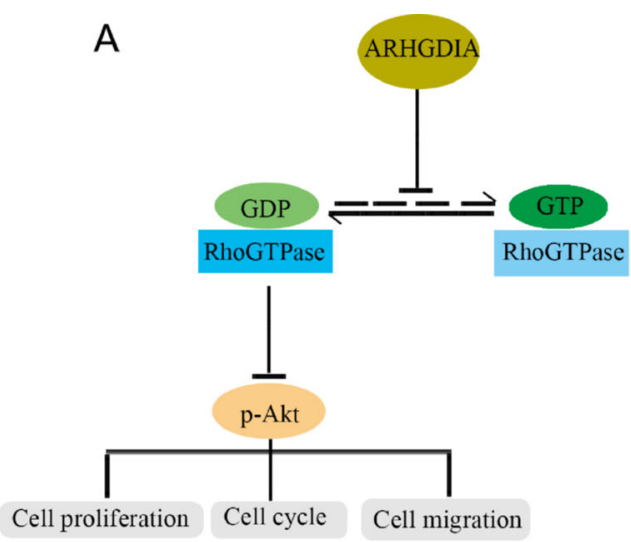

Fig. 7 Schematic diagram summary of ARHGDIA effects on tumor progression in human gliomas. Under normal physiological conditions, ARHGDIA inhibits activation of Rho proteins by maintaining the GDPbound Rho proteins in cytosol (a). When ARHGDIA protein is
Rac1, and RhoA, subsequently, increases Akt phosphorylation and leads to glioma cell proliferation and migration.

\section{Discussion}

Numerous studies indicate that GTPase signaling pathway closely correlates with tumors [16], and the expression of RhoGDIs which are the regulators of the Rho family of small GTPases is altered in different cancers. For instance, ARHGDIA expression is upregulated in colorectal [17] and ovarian cancers [18]. However, a significant reduction of

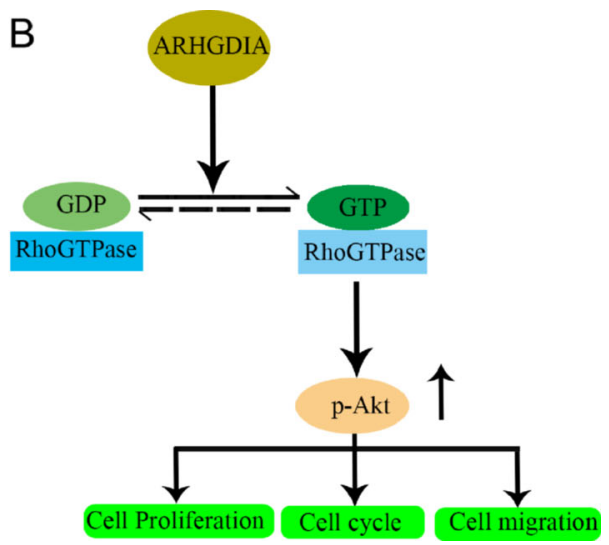

downregulated in glioma, the GTP-form Rho GTPases are activated (b) and subsequently Akt phosphorylaton is elevated, which promotes cell proliferation, cell cycle, and cell migration 
ARHGDIA expression is detected in breast cancer [6]. In addition, ARHGDIA downregulation is associated with poor prognosis in hepatocellular carcinoma [7]. These different expression profiling even opposite results on this protein in several cancers urgently drives us to know how ARHGDIA mediates the processes during tumorigenesis and cancer progression in human glioma.

It has been reported ARHGDIA interacts with different proteins to involve in cell motility. ARHGDIA can interact with $\alpha v \beta 8$ integrin, and ARHGDIA- $\alpha v \beta 8$ integrin protein complexes recruit GDP-bound Rac1 and $\mathrm{Cdc} 42$ to control activation of Rho proteins. When silencing ARHGDIA gene expression leads to elevated levels of GTP-bound Rho proteins, which results in diminishing cell polarity and invasion [19]. Moreover, phosphorylation of ARHGDIA on Y156 leads to deposition of Rac1/Cdc42 proteins and enabling their activation to promote directional cell motility [20]. These indicate that the ARHGDIA protein affects the activation of Rho proteins mainly via coupling other proteins such as $\alpha v \beta 8$ integrin. On the other hand, although ARHGDIA has been reported to decrease in brain tumors before [21], but there could not clearly clarify which member of RhoGDI family to change and its specific biological effects. In the present study, we further investigated the role of ARHGDIA in human glioma, and we firstly discovered the relationship between the dysregulation of ARHGDIA and glioma progression. Our research showed that ARHGDIA is frequently down-regulated in human glioma and significantly correlates with prognosis of glioma patients.

As the regulator of Rho GTPases family, we further focus on the changes of Rho GTPase proteins after ARHGDIA knockdown in human glioma cells. Previous researches had indicated that downregulation of ARHGDIA had a different effect on the activation of Rho GTPase family members in different tumor types. For example, Turner et al. found that the member of RhoGTPase was increased significantly in the HeLa cells transfected with ARHGDIA siRNA [22]. In myocardial cells, overexpression of ARHGDIA significantly inhibits the activities of Rac1, Cdc42, and RhoA [23]. But in ARHGDIA-knockout mice, renal abnormality is just associated with an increase of Rac1 but not RhoA [24], whereas loss of ARHGDIA significantly induced the activation of Rac1, RhoA but not Cdc42 in HCC [7]. By now, we found that knockdown of ARHGDIA by siRNA resulted in activating the GTPase activity of Cdc42, Rac1, RhoA, and pAkt to promote glioma cell proliferation and migration. To the best of our knowledge, this is the first study to discover the downregulation of ARHGDIA with glioma tumor progression by multiple biochemical analyses, overexpression, and knockdown in vitro combined with clinical sample validation.

Akt acts downstream of Rac1 and Cdc42 in the control of cellular survival [15], which indicates that Akt signaling is closely related to Rho GTPase signaling pathway. And our data supported that the activation of GTPase activity increases the downstream phosphorylated Akt signaling, which finally induces cell proliferation and cell cycle progression in human glioma cells. So based on the results of our research and literature reported, the potential action mechanism of ARHGDIA in glioma is summarized as following (Fig. 7). Under cell normal physiological conditions, ARHGDIA level makes a balance state of the GDP-bound inactive form and GTPbound GTPase form of Rho family proteins (Fig. 7a). While the low expression of ARHGDIA in human glioma attenuates the inhibition effects of the GDP-bound inactive Rho family protein, and the GTP-bound form of Rho GTPases (Cdc42, Rac1, and Rho) is activated to switch on downstream pathways by acting on theirs effectors including improving phosphorylated Akt (Fig. 7b), which finally promotes cancer biological process including cell proliferation, cell cycle progression, and cell migration.

ARHGDIA, Rho GDP-dissociation inhibitor 1; HGTs: human glioma tissues; $I H C$, immunohistochemistry; $P B T$ s, paracancerous brain tissues; siRNA, small interfering RNA

Acknowledgments This work was financially supported by the grants from National Key Basic Research Program of China (2013CB911303, 2011CB910703), National 863 High Tech Foundation (2014AA020608), National Natural Sciences Foundation of China (31470810, 31071235, 30970654), and the Doctoral Program of Higher Education (20120181110025).

Authors' contributions Lu W carried out experiments and drafted the manuscript. Wang X, Liu J, He Y, Liang Z, Cai Y, and Xia Z performed experiments. Zhou L and Zhu H analyzed clinical samples. Liang S supervised the study and revised manuscript. All authors read and approved the final manuscript.

\section{Compliance with ethical standard}

Conflicts of interest None

Open Access This article is distributed under the terms of the Creative Commons Attribution 4.0 International License (http:// creativecommons.org/licenses/by/4.0/), which permits unrestricted use, distribution, and reproduction in any medium, provided you give appropriate credit to the original author(s) and the source, provide a link to the Creative Commons license, and indicate if changes were made.

\section{References}

1. Ostrom QT, Gittleman H, Farah P, Ondracek A, Chen Y, Wolinsky $\mathrm{Y}$, et al. Cbtrus statistical report: primary brain and central nervous system tumors diagnosed in the United States in 2006-2010. NeuroOncology. 2013;15 Suppl 2:ii1-56.

2. Louis DN, Ohgaki H, Wiestler OD, Cavenee WK, Burger PC, Jouvet A, et al. The 2007 who classification of tumours of the central nervous system. Acta Neuropathol. 2007;114:97-109. 
3. Wen PY, Kesari S. Malignant gliomas in adults. N Engl J Med. 2008;359:492-507.

4. Liang S, Shen G. Biomarkers of glioma. INTECH Open Access Publisher, 2011.

5. Cherfils J, Zeghouf M. Regulation of small gtpases by gefs, gaps, and gdis. Physiol Rev. 2013;93:269-309.

6. Jiang WG, Watkins G, Lane J, Cunnick GH, Douglas-Jones A, Mokbel K, et al. Prognostic value of rho gtpases and rho guanine nucleotide dissociation inhibitors in human breast cancers. Clin Cancer Res. 2003;9:6432-40.

7. Liang L, Li Q, Huang L, Li D, Wang Y, Li X, et al. Loss of arhgdia expression is associated with poor prognosis in hec and promotes invasion and metastasis of hec cells. Int J Oncol. 2014.

8. Harding MA, Theodorescu D. Rhogdi signaling provides targets for cancer therapy. Eur J Cancer. 2010;46:1252-9.

9. Hoffman GR, Nassar N, Cerione RA. Structure of the rho family gtp-binding protein cdc42 in complex with the multifunctional regulator rhogdi. Cell. 2000;100:345-56.

10. Liang S, Xu Y, Shen G, Zhao X, Zhou J, Li X, et al. Gene expression and methylation status of 14-3-3 $\sigma$ in human renal carcinoma tissues. IUBMB Life. 2008;60:534-40.

11. Jin X, Liu Y, Liu J, Lu W, Liang Z, Zhang D, et al. The overexpression of iqgap1 and $\beta$-catenin is associated with tumor progression in hepatocellular carcinoma in vitro and in vivo. PLoS One. 2015;10:e133770.

12. Zhang LH, Yin AA, Cheng JX, Huang HY, Li XM, Zhang YQ, et al. Trim 24 promotes glioma progression and enhances chemoresistance through activation of the pi3k/akt signaling pathway. Oncogene. 2014;34:600-10.

13. Boulter E, Garcia-Mata R, Guilluy C, Dubash A, Rossi G, Brennwald PJ, et al. Regulation of rho gtpase crosstalk, degradation and activity by rhogdil. Nat Cell Biol. 2010;12:477-83.

14. Ponomarev I, Wang S, Zhang L, Harris RA, Mayfield RD. Gene coexpression networks in human brain identify epigenetic modifications in alcohol dependence. J Neurosci. 2012;32:1884-97.
15. Zugasti O, Rul W, Roux P, Peyssonnaux C, Eychene A, Franke TF, et al. Raf-mek-erk cascade in anoikis is controlled by rac1 and cdc42 via akt. Mol Cell Biol. 2001;21:6706-17.

16. Goldberg L, Kloog Y. A ras inhibitor tilts the balance between rac and rho and blocks phosphatidylinositol 3-kinase-dependent glioblastoma cell migration. Cancer Res. 2006;66:11709-17.

17. Zhao L, Wang H, Sun X, Ding Y. Comparative proteomic analysis identifies proteins associated with the development and progression of colorectal carcinoma. FEBS J. 2010;277:4195-204.

18. Jones MB, Krutzsch H, Shu H, Zhao Y, Liotta LA, Kohn EC, et al. Proteomic analysis and identification of new biomarkers and therapeutic targets for invasive ovarian cancer. Proteomics. 2002;2:7684.

19. Reyes SB, Narayanan AS, Lee HS, Tchaicha JH, Aldape KD, Lang $\mathrm{FF}$, et al. Alphavbeta8 integrin interacts with rhogdi1 to regulate rac1 and cdc42 activation and drive glioblastoma cell invasion. Mol Biol Cell. 2013;24:474-82.

20. Lee HS, Cheerathodi M, Chaki SP, Reyes SB, Zheng Y, Lu Z, et al. Protein tyrosine phosphatase-pest and beta8 integrin regulate spatiotemporal patterns of rhogdil activation in migrating cells. Mol Cell Biol. 2015;35:1401-13.

21. Forget M-A, Desrosiers RR, Del Maestro RF, Moumdjian R, Shedid D, Berthelet F, et al. The expression of rho proteins decreases with human brain tumor progression: potential tumor markers. Clin Exp Metastasis. 2002;19:9-15.

22. Turner SJ, Zhuang S, Zhang T, Boss GR, Pilz RB. Effects of lovastatin on rho isoform expression, activity, and association with guanine nucleotide dissociation inhibitors. Biochem Pharmacol. 2008;75:405-13.

23. Wei L, Imanaka-Yoshida K, Wang L, Zhan S, Schneider MD, DeMayo FJ, et al. Inhibition of rho family gtpases by rho gdp dissociation inhibitor disrupts cardiac morphogenesis and inhibits cardiomyocyte proliferation. Development. 2002;129:1705-14.

24. Shibata S, Nagase M, Yoshida S, Kawarazaki W, Kurihara H, Tanaka H, et al. Modification of mineralocorticoid receptor function by rac1 gtpase: implication in proteinuric kidney disease. Nat Med. 2008;14:1370-6. 